\title{
Cancellation of simple optical anisotropies without use of a Faraday mirror
}

\author{
Rajendra Bhandari \\ Raman Research Institute, \\ Bangalore 560 080, India. \\ email: bhandari@rri.res.in
}

\begin{abstract}
We first derive the round-trip Jones matrix for double passage through a reciprocal optical medium by means of reflection off a plane mirror that could be optically anisotropic. We then show that if a medium with only linear birefringence and linear dichroism is placed between a pair of orthogonal quarterwave plates with principal axes at $45^{\circ}$ to that of the medium and the sandwich is placed in front of an isotropic mirror it behaves, under double passage, like an isotropic medium. We describe a simple liquid crystal device that behaves, in reflection, as an isotropic medium whose refractive index can be varied by application of an electric field, thus acting as a phase only modulator for light in any polarization state.
\end{abstract}

(c) 2021 Optical Society of America

OCIS codes: 260.5430, 230.5440, 230.6120, 230.3720

When a monochromatic plane polarized light wave changes its direction of propagation in space either by means of reflection or by means of refraction, the description of the polarization state in the new direction involves the choice of a new set of basis states along with a new set of phases for these basis states. Definition of a round-trip Jones matrix for double passage through a medium requires a clear statement of these choices. A natural choice is the following: Take a set of linearly polarized vibrations in and perpendicular to a chosen plane of incidence ( $\mathrm{p}$ and s states), say along $\hat{x}$ and $\hat{y}$, vibrating in phase, as the basis states for the original direction of propagation. Rotate the two linear vibrations about an axis perpendicular to the plane of incidence by an angle equal to that between the final and initial directions so that $\hat{x}$ and $\hat{y}$ go to $\hat{x}^{\prime}$ and $\hat{y}^{\prime}$ respectively. A set of linear vibrations along $\hat{x}^{\prime}$ and $\hat{y}^{\prime}$ in phase with each other then provides a natural choice for the basis states as well as their phases, for the new direction of propagation. We shall call this the "travelling frame 
convention". This is the convention used to write the Fresnel's relations for reflection and refraction at an interface for arbitrary angles of incidence [1] and to define the reflection Jones matrix in ellipsometry. Considerations in [2] also support the above choice. The reflection Jones matrix $Z$ acting on the initial Jones vector $\mid i>$ gives a new vector $\mid f>$ whose components represent the amplitudes with respect to the new base states.

Therefore let the polarization basis states for the initial direction of propagation be chosen to be (i) $E_{x}=E \exp (i \omega t), E_{y}=0$ and (ii) $E_{x}=0, E_{y}=E \exp (i \omega t)$, where $E_{x}$ and $E_{y}$ are the $x$ and $y$ components of the electric field in the wave. Let the initial Jones vector be $\mid i>=$ col. $\left[c_{1}, c_{2}\right]$. The fields in the initial wave are then $E_{x}=c_{1} E \exp (i \omega t), \quad E_{y}=$ $c_{2} E \exp (i \omega t)$. In the above stated convention the basis states for the final direction of propagation are (i) $E_{x^{\prime}}=E \exp (i \omega t), E_{y^{\prime}}=0$ and (ii) $E_{x^{\prime}}=0, E_{y^{\prime}}=E \exp (i \omega t)$. If $|f>=Z| i>=\operatorname{col} .\left[c_{1}^{\prime}, c_{2}^{\prime}\right]$ is the final Jones vector, where $Z$ is a Jones matrix, the fields in the final wave are given by $E_{x^{\prime}}=c_{1}^{\prime} E \exp (i \omega t), \quad E_{y^{\prime}}=c_{2}^{\prime} E \exp (i \omega t)$.

We next use the method described in $[2,3]$ to derive a round trip Jones matrix for double passage through a reciprocal optical medium $M$ by means of reflection off an optically anisotropic mirror (Fig.1(a)). In this Letter we shall ignore all constant isotropic phase factors equal to $i$ and -1 . Let $\hat{z}$ and $-\hat{z}$ be the two directions of propagation and let the reflection plane be chosen to be the $(\hat{x}, \hat{z})$ plane [4]. Following [2,3], the actual optical circuit shown in Fig.1(a) can be replaced by an equivalent circuit shown in Fig.1(b) where the mirror has been replaced by the reflection matrix $Z$ of the surface determined with the above convention and the medium $M$ during reverse passage has been replaced by $\bar{M}$, obtained from $M$ by rotation about $\hat{y}$ through $\pi$. It was shown in [5] that

$$
\bar{M}=\left(\begin{array}{lr}
m_{11} & -m_{21} \\
-m_{12} & m_{22}
\end{array}\right) \text { for } M=\left(\begin{array}{ll}
m_{11} & m_{12} \\
m_{21} & m_{22}
\end{array}\right) .
$$

As shown in Fig.1(b), the equivalent evolution consists of two parts: (a) a pure polarization evolution given by a product matrix $M^{(\mathrm{rt})}$ that we shall call the round trip Jones matrix and (b) a $\pi$ rotation of the beam about $\hat{y}$. The operation (b) does not affect the ellipticity of the polarization ellipse but does change its absolute orientation in space. The round trip Jones matrix $M^{(\mathrm{rt})}$ is therefore given by,

$$
M^{(\mathrm{rt})}=\bar{M} Z M
$$

If $\left|f>=M^{(\mathrm{rt})}\right| i>=\operatorname{col}\left[\left[c_{1}^{\prime}, c_{2}^{\prime}\right]\right.$ is the final Jones vector, the fields resulting from operation (A) are $E_{x}=c_{1}^{\prime} E \exp (i \omega t), E_{y}=c_{2}^{\prime} E \exp (i \omega t)$, while those after taking into account both operations (A) and (B) are $E_{x^{\prime}}=c_{1}^{\prime} E \exp (i \omega t), E_{y^{\prime}}=c_{2}^{\prime} E \exp (i \omega t)$, where $\hat{x}^{\prime}=-\hat{x}$ and $\hat{y}^{\prime}=$ $\hat{y}$. 


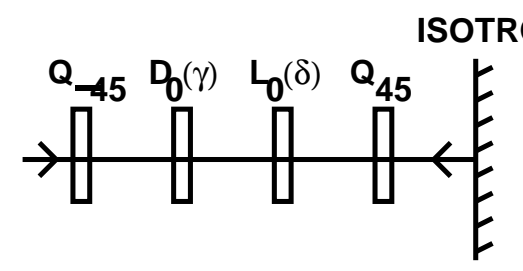

(a)

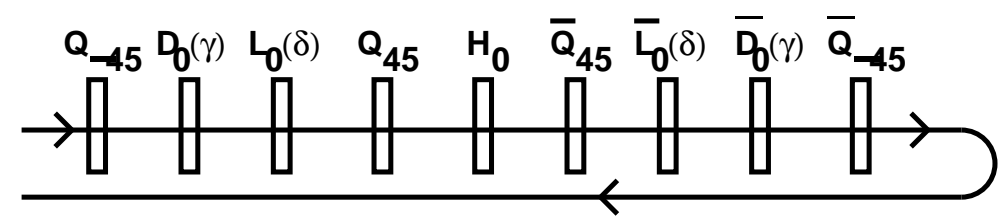

(b)

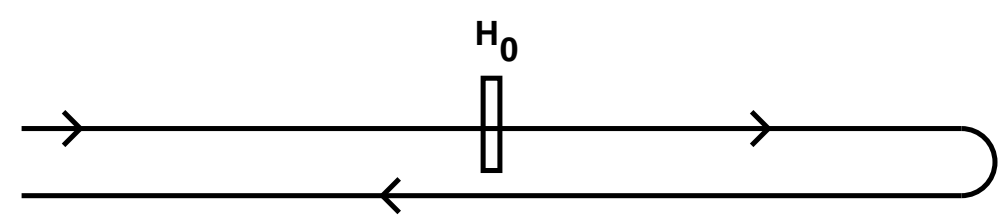

(c)

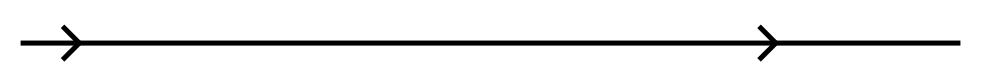

(d)

Fig. 1. A sandwich consisting of a reciprocal optical medium with linear birefringence and linear dichroism along the same axis, placed between a pair of orthogonal quarterwave plates at $+45^{\circ}$ or $-45^{\circ}$ behaves like an isotropic medium when placed in front of an isotropic mirror. (a) The actual optical configuration, (b) the equivalent optical circuit, (c) the reduced circuit and (d) an equivalent reduced circuit in the fixed frame. The isotropic factors $a, p$ and $r$ have been omitted for convenience. 
While this completes the description of our method, it is worthwhile to take note of another useful convention often used in the analysis of double passage problems. In this convention, which holds only for normal incidence, both the initial and the return Jones vectors are expressed in the same i.e. the initial set of basis states. In other words a given polarization ellipse in space is represented by the same Jones vector irrespective of whether the beam is travelling forward or backward [6]. The Jones matrix describes the evolution of this ellipse. We shall call this the "fixed frame convention". Since the only change involved is the sign of the $x$-component of the electric field in the final wave, it is equivalent to the action of a half-wave plate $H_{0}$ where $H_{0}$ is a half-wave plate with its fast axis along $\hat{y}$. One can therefore describe the evolution of the polarization ellipse in the fixed frame as shown schematically in Fig.1(c) in terms of a matrix $M^{\prime(\mathrm{rt})}$ given by

$$
M^{\prime(\mathrm{rt})}=H_{0} \bar{M} Z M
$$

When the mirror is optically isotropic, $Z=r H_{0}$ where $r$ is a constant isotropic attenuation factor representing the reflectivity of the surface [7]. For this case Eqns. (2) and (3) are replaced by, $M^{(\mathrm{rt})}=r \bar{M} H_{0} M$ and $M^{\prime(\mathrm{rt})}=r H_{0} \bar{M} H_{0} M$.

It is well known that double passage of a polarized light beam through a reciprocal medium by reflection off a Faraday mirror can cancel an arbitrary optical anisotropy in the medium $[5,8,9]$. In the following we first show that if a reciprocal medium that has only linear birefringence or linear dichroism or both, with the same principal axes, is placed between a pair of orthogonal quarter-wave plates with principal axes at $+45^{\circ}$ or $-45^{\circ}$ to that of the medium and the sandwich is placed in front of an isotropic mirror as shown in Fig.2(a), it behaves, under double passage, like an isotropic medium placed in front of a mirror. The following matrix identities will be useful in proving the result.

$$
\begin{aligned}
& L_{\phi}(\delta)=R(\phi) L_{0}(\delta) R(-\phi), D_{\phi}(\gamma)=R(\phi) D_{0}(\gamma) R(-\phi), \\
& \bar{L}_{\phi}(\delta)=L_{-\phi}(\delta), \bar{D}_{\phi}(\gamma)=D_{-\phi}(\gamma), \\
& D_{\phi}(\gamma) D_{\phi+90^{\circ}}(\gamma)=L_{\phi}(\delta) L_{\phi+90^{\circ}}(\delta)=\mathbf{1}, \\
& R\left(\phi_{1}\right) R\left(\phi_{2}\right)=R\left(\phi_{1}+\phi_{2}\right), R( \pm 180)=-\mathbf{1} \\
& H_{\phi}=L_{\phi}(\pi), Q_{\phi}=L_{\phi}(\pi / 2), Q_{\phi} H_{\phi} Q_{\phi}=-\mathbf{1} \\
& \text { and } R(\phi) H_{0} R(\phi)=H_{0},
\end{aligned}
$$

where $L_{\phi}(\delta)$ stands for a linear retarder with retardation $\delta$ and with its fast axis making an angle $\phi$ with $\hat{y}, D_{\phi}(\gamma)$ is a linearly dichroic element with relative attenuation coefficient $\gamma$ and its easy axis at an angle $\phi$ with $\hat{y}$ and $R(\phi)$ is an optical rotator with rotation $\phi$ in real space, all three being elements of the Special Linear group $\mathrm{SL}(2, \mathrm{C})$. If the principal axes of 


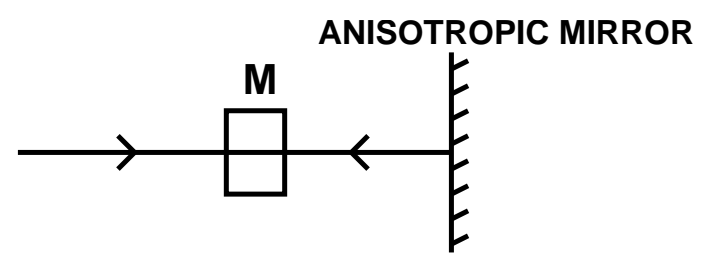

(a)

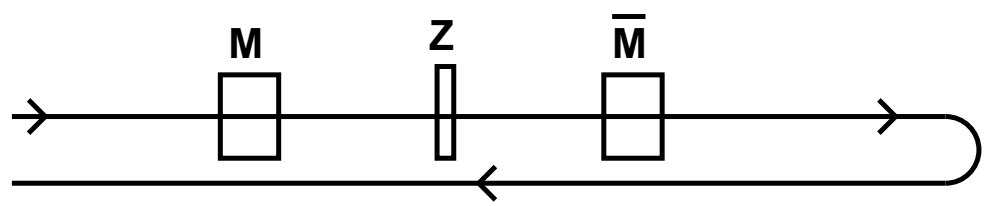

(b)

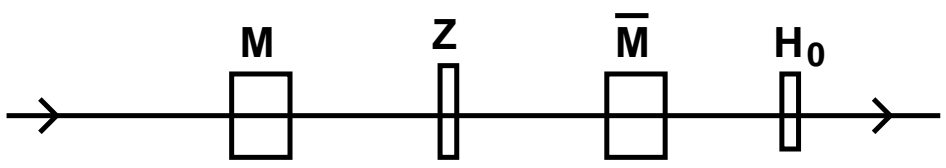

(c)

Fig. 2. (a) Double passage of a polarized light beam through a reciprocal medium by means of normal reflection off an optically anisotropic surface, (b) the equivalent optical circuit consisting of a pure polarization evolution given by the Jones matrix $\bar{M} Z M$ followed by a rotation of the beam about an axis perpendicular to the plane of incidence and (c) an equivalent circuit in the fixed frame for the evolution shown in (b). 
the medium are aligned to be along $\hat{x}$ and $\hat{y}$, the Jones matrix $M$ of the medium for single passage is given by,

$$
M=a p L_{0}(\delta) D_{0}(\gamma),
$$

where $a$ and $p$ are the isotropic absorption and phase factors for single passage. The equivalent optical circuit for double passage is shown in Fig.2(b). The round trip Jones matrix $M^{(\mathrm{rt})}$ for the polarization evolution is given by,

$$
\begin{aligned}
& M^{(\mathrm{rt})}=r a^{2} p^{2} \bar{Q}_{-45} \bar{D}_{0}(\gamma) \bar{L}_{0}(\delta) \bar{Q}_{45} H_{0} Q_{45} L_{0}(\delta) D_{0}(\gamma) Q_{-45} \\
& =r a^{2} p^{2} Q_{45} D_{0}(\gamma) L_{0}(\delta) Q_{-45} H_{0} Q_{45} L_{0}(\delta) D_{0}(\gamma) Q_{-45} \\
& =r a^{2} p^{2} R(45) Q_{0} R(-45) D_{0}(\gamma) L_{0}(\delta) R(-45) Q_{0} R(45) H_{0} \\
& \times R(45) Q_{0} R(-45) L_{0}(\delta) D_{0}(\gamma) R(-45) Q_{0} R(45) \\
& =-r a^{2} p^{2} R(45) Q_{0} R(-45) D_{0}(\gamma) L_{0}(\delta) R(-90) L_{0}(\delta) D_{0}(\gamma) \\
& \times R(-45) Q_{0} R(45) \\
& =-r a^{2} p^{2} R(45) Q_{0} R(-45) R(-90) R(90) D_{0}(\gamma) L_{0}(\delta) R(-90) \\
& \times L_{0}(\delta) D_{0}(\gamma) R(-45) Q_{0} R(45) \\
& =-r a^{2} p^{2} R(45) Q_{0} R(-45) R(-90) D_{90}(\gamma) L_{90}(\delta) L_{0}(\delta) D_{0}(\gamma) \\
& \times R(-45) Q_{0} R(45) \\
& =-r a^{2} p^{2} R(45) Q_{0} R(-180) Q_{0} R(45) \\
& =r a^{2} p^{2} H_{0} .
\end{aligned}
$$

where one or more of the identities (44) - (9) have been used in each step of simplification. Fig.2(c) shows the final result. $M^{\prime(\mathrm{rt})}$ is given by,

$$
M^{\prime(\mathrm{rt})}=H_{0} M^{(\mathrm{rt})}=r a^{2} p^{2} \mathbf{1} .
$$

This is shown in Fig.2(d). Equations.(11) and (12) together with Figs. 2(c) and 2(d) demonstrate the equivalence of the device with an isotropic medium placed in front of an isotropic mirror (compare with Fig.1 with $M=1, Z=H_{0}$ ). Since $M^{(\mathrm{rt})}$ and $M^{\prime(\mathrm{rt})}$ are independent of $\delta$ and $\gamma$, the anisotropies have effectively been cancelled. The isotropic factors $a, p$ and $r$ however survive. A similar result is obtained if $Q_{ \pm 45}$ is replaced by $Q_{\mp 45}$.

Using a similar analysis it can be shown that if an optical element with circular dichroism and optical activity, which can be expressed as a product $Q_{ \pm 45} D_{0}(\gamma) Q_{\mp 45} R(\phi)$, is placed in front of an isotropic mirror, the round trip matrix for double passage is the same as that of 
an isotropic medium placed in front of the mirror. Optical activity and circular dichroism can therefore be cancelled just by reflection off an isotropic mirror. We also point out that in the above two examples, since $M^{\prime(\mathrm{rt})}=1$, the polarization ellipse is unchanged in the fixed frame as in the case of an isotropic medium. In the Faraday mirror cancellation, however, $M^{\prime(\mathrm{rt})}=R( \pm 90)$ and the polarization ellipse gets rotated through $90^{\circ}$.

We next present a practical application of cancellation of linear birefringence. A transparent linearly birefringent substance can be characterized by $\gamma=0$ (or $a=1$ ) and two refractive indices $n_{e}$ and $n_{o}$ representing the velocities of propagation for electric vectors along the two principal axes that can be aligned to be along $\hat{x}$ and $\hat{y}$. The isotropic phase factor $p$ is given by,

$$
p=\exp \left(i \phi_{\text {iso }}\right) \text { with } \phi_{\text {iso }}=(2 \pi d / \lambda)\left(n_{e}+n_{o}\right) / 2
$$

where $d$ is the thickness of the sample and $\lambda$ is the wavelength of light. The retardation $\delta$ due to birefringence is given by

$$
\delta=(2 \pi d / \lambda)\left(n_{e}-n_{o}\right)
$$

Any material in which $\phi_{\text {iso }}$ given by Eqn.(13) can be varied using an electric field, placed between two orthogonal quarterwave plates and the sandwich placed in front of a mirror as described above acts as an isotropic medium with its refractive index adjustable by means of an electric field. An example of such a material is a suitably prepared nematic liquid crystal cell in which $n_{e}=<n_{e}(z)>$ varies with an applied electric field and $n_{o}$ remains constant [10]. The two quarterwave plates could in fact form part of the liquid crystal cell itself. Such a device would act as a 'phase only' modulator for light in any polarization state. A closely related device for modulating unpolarized light using a nematic liquid crystal cell and a $45^{\circ}$ quarterwave plate placed in front of a mirror has earlier been described by Love [11].

In this Letter we have ignored constant isotropic phase factors equal to $i$ and -1 . This is justified in problems where one is interested only in phase changes. However the applications described in this Letter and in [5] demonstrate that throwing away the isotropic factors altogether may at times be like "throwing away the baby with the bath". The device described in this Letter in fact works because $\phi_{i s o}$ can be controlled using an electric field. We also note that the analysis in this paper applies only in the regime of linear optics and not to double passage caused by optical phase conjugation.

Another possible application of the method described in this Letter is in the analysis of Michelson interferometers where the beam splitter and the retroreflecting mirrors have optical anisotropy and polarization changing elements have been placed in one or both paths 
of the interferometer. In this application since one compares two beams travelling in the same direction, if the interferometer is planar, the second part of the evolution shown in Fig.1(b) can be ignored and the matrix $M^{(\mathrm{rt})}$, computed separately for each of the two paths, provides a complete solution to the interference problem. The beam splitter reflection and the mirror reflection at normal incidence are treated similarly in this method, i.e. both are represented by their natural $Z$-matrix.

\section{References}

1. M. Born and E. Wolf, "Principles of optics", (Pergamon 1975), p.40.

2. R. Bhandari, "Geometric phase in an arbitrary evolution of a light beam," Phys. Lett. A 135, 240-244 (1989) .

3. R. Bhandari, "Evolution of light beams in polarization and direction," Physica B 175, 111-122 (1991).

4. It can be shown that the final results of any calculation by this method is independent of the choice of the plane of incidence.

5. R. Bhandari, "Transpose symmetry of the Jones matrix and topological phases," Opt. Lett. 33 , 854-856 (2008).

6. R. C. Jones, "A new calculus for the treatment of optical systems I. Description and discussion of the calculus," J. Opt. Soc. Am. 31, 488-493 (1941).

7. The factor $r$, though unimportant, should also have been present in the analysis described in $[5]$.

8. N.C. Pistoni and M. Martinelli, "Polarization noise suppression in retracing optical fiber circuits," Opt. Lett. 16, 711-713 (1991).

9. M. O. van Deventer, "Preservation of polarization orthogonality of counterpropagating waves through dichroic birefringent media: proof and application," Electron. Lett. 27, 1538-1539 (1991).

10. V. G. Chigrinov, "Liquid crystal devices: physics and application", (Artech 1999), p.85.

11. G. D. Love, "Liquid-crystal phase modulator for unpolarized light", App. Opt. 32, 22222223 (1993). 